\title{
Richtgrößen
}

\section{Ruhestand schützt nicht vor Regress}

\author{
Ärzte müssen auch noch unmittelbar vor dem Ruhestand auf \\ Einhaltung ihrer Richtgrößen achten. In zwei Urteilen präzisiert \\ das Bundessozialgericht diese Rechtslage nun.
}

$\ddot{A}$ rzte müssen auch noch kurz vor ihrem Ruhestand darauf achten, dass sie ihre Richtgrößen einhalten. Ein Regress wegen Überschreitung der Richtgrößen kann auch noch nach dem Ausscheiden aus der vertragsärztlichen Versorgung festgesetzt werden. Das bestätigte kürzlich das Bundessozialgericht (BSG). Nach einem weiteren Urteil mindern entsprechende Regressforderungen auch die Gesamtvergütung (BSG Az.: B 6 KA 45/14 R [Regress] und B 6 KA 15/15 R [Gesamtvergütung]).

Im ersten Fall hatte ein Arzt in Schleswig-Holstein von 2003 bis 2005 sein Richtgrößenvolumen quartalsweise um bis zu $97 \%$ überschritten. 2006 verzichtete er auf seine Zulassung und schied aus der vertragsärztlichen Versorgung aus. Der Prüfungsausschuss meinte daher, ein Regress sei nicht mehr möglich.

Auf den Widerspruch der Kassenverbände setzte der Beschwerdeausschuss dann 144.300 Euro Regresssumme fest. Die Klage des Arztes blieb durch alle Instanzen ohne Erfolg. „Ein Regress wegen Überschreitung der Richtgrößen kann gegen den Vertragsarzt auch noch nach dessen Ausscheiden aus der vertragsärztlichen Versorgung festgesetzt werden", urteilte das BSG. Entsprechend hatten Kasseler Richter 2013 bereits in der Angelegenheit eines Zahnarztes entschieden.

Zur Begründung erklärten die Richter, ein Arzt bleibe auch nach Eintritt in den Ruhestand für seine frühere Behandlungs- und Verordnungsweise verantwortlich. Das werde auch nicht dadurch infrage gestellt, dass ein aus dem Dienst geschiedener Arzt den Regress nicht mehr durch eine für die Zukunft gültige Richtgrößenvereinbarung abwenden kann. Dass die KV nicht mit späteren
Honoraren aufrechnen kann, stehe dem Regress ebenfalls nicht entgegen.

Allerdings habe es der Beschwerdeausschuss diesbezüglich versäumt, dem Arzt eine freiwillige Vereinbarung über den Regress anzubieten. Laut Gesetz soll die Prüfungsstelle auf solch eine Vereinbarung „hinwirken“.

\section{Gesamtvergütung wird gekürzt}

Wenn der Arzt darin die Forderung anerkennt, können im Gegenzug bis zu 20 $\%$ der Forderung erlassen werden. Das BSG hatte im Juli 2015 entschieden, dass die Regressforderung pauschal um $20 \%$ zu kürzen ist, wenn - wie hier - eine solche Vereinbarung nun nicht mehr möglich ist (Az.: B 6 KA 0/14 R).
In einem weiteren Urteil entschied das BSG zudem, dass auch bei einem nach Ausscheiden des Arztes festgesetzten Regress die Gesamtvergütung entsprechend zu kürzen ist. Die gesetzlichen Regelungen sähen hier keine Ausnahme vor. Danach sei die Gesamtvergütung sogar dann zu kürzen, wenn die Regressforderung etwa wegen Insolvenz nicht mehr eingetrieben werden kann. Das Argument, dies sei quasi eine Rückkehr zu dem bis 2001 geltenden und dann gesetzlich beendeten „Kollektivregress“, ließ der BSG-Senat nicht gelten. Hier knüpfe die Kürzung der Gesamtvergütung an das Verhalten einzelner Ärzte an und nicht wie damals an das der Vertragsärzte insgesamt. Zudem wirke sich die Kürzung für andere Ärzte nur dann aus, „wenn die KV die Regressforderung gegenüber dem Arzt ausnahmsweise nicht durchzusetzen vermag“. Dieses Risiko müsse die KV tragen, so die Richter.

Martin Wortmann

\section{Hier steht eine Anzeige.}

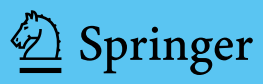

